\title{
An insight into the public acceptance of nutrigenomic-based personalised nutrition
}

\author{
R. Fallaize ${ }^{1}$, A. L. Macready ${ }^{1}$, L. T. Butler ${ }^{2}$, J. A. Ellis ${ }^{2}$ and J. A. Lovegrove ${ }^{1 *}$ \\ ${ }^{1}$ Department of Food and Nutritional Sciences and Institute for Cardiovascular and Metabolic Research (ICMR), \\ University of Reading, Whiteknights, Reading RG6 6AP, UK \\ ${ }^{2}$ Department of Psychology, University of Reading, Earley Gate, Whiteknights, Reading RG6 GAL, UK
}

\section{Abstract}

It is predicted that non-communicable diseases will account for over $73 \%$ of global mortality in 2020 . Given that the majority of these deaths occur in developed countries such as the UK, and that up to $80 \%$ of chronic disease could be prevented through improvements in diet and lifestyle, it is imperative that dietary guidelines and disease prevention strategies are reviewed in order to improve their efficacy. Since the completion of the human genome project our understanding of complex interactions between environmental factors such as diet and genes has progressed considerably, as has the potential to individualise diets using dietary, phenotypic and genotypic data. Thus, there is an ambition for dietary interventions to move away from population-based guidance towards 'personalised nutrition'. The present paper reviews current evidence for the public acceptance of genetic testing and personalised nutrition in disease prevention. Health and clear consumer benefits have been identified as key motivators in the uptake of genetic testing, with individuals reporting personal experience of disease, such as those with specific symptoms, being more willing to undergo genetic testing for the purpose of personalised nutrition. This greater perceived susceptibility to disease may also improve motivation to change behaviour which is a key barrier in the success of any nutrition intervention. Several consumer concerns have been identified in the literature which should be addressed before the introduction of a nutrigenomic-based personalised nutrition service. Future research should focus on the efficacy and implementation of nutrigenomic-based personalised nutrition

Key words: Personalised nutrition: Consumer acceptance: Disease prevention: Nutrigenomics

\section{Introduction}

The need for targeted prevention of key non-communicable diseases (NCD) such as CVD and diabetes is a priority in the UK as NCD are expected to account for over $73 \%$ of global mortality in $2020^{(1)}$. CVD accounts for over half of the deaths caused by NCD and is the main cause of mortality in the European Union, contributing to over 2 million deaths each year. In 2008, CVD accounted for more than $30 \%$ of all deaths in the UK, with total health care costs reaching in excess of $£ 18$ billion $^{(2)}$. CVD are chronic diseases with a long latency period and a spectrum of risk factors. Advances in research have led to the identification of many reliable biochemical and clinical indicators that have enabled earlier identification of individuals at risk of CVD and, hence, increased opportunities for preventative interventions. NCD are polygenic diseases involving complex interactions between two or more genes and the environment. Unhealthy diets and physical inactivity are amongst the key causes of NCD and must be addressed in order to reduce the significant impact that they have on mortality and morbidity; it is estimated that about $80 \%$ of CHD and cerebrovascular disease could be avoided by improvements in lifestyle choices such as healthier food consumption ${ }^{(3)}$. Given that personalised health communication has been found to be more effective in stimulating greater cognitive activity, it has become increasingly clear that dietary guidance may need to move away from generic guidance, towards more tailored disease prevention strategies ${ }^{(4)}$.

Current nutrient intake guidelines in the UK are based on population estimates of the macro- and micronutrient intake required to prevent malnourishment. In some cases requirements are separated according to age, sex and key physiological states such as pregnancy. Individuals identified to be at high risk of disease through assessments, such as the QRISK ${ }^{\circledR}$ score for CVD, typically have access to more individualised advice ${ }^{(5,6)}$ often based on the

Abbreviations: DTC, direct-to-consumer; HCP, health care professional; NCD, non-communicable disease; PGT, personal genome testing.

*Corresponding author: Professor Julie Lovegrove, email J.A.Lovegrove@reading.ac.uk 
pathophysiology of the disease and identified population characteristics of diseased individuals. However, it is known that individuals respond differently to nutrients with varying levels of individual benefit and risk observed at given doses ${ }^{(7)}$. Whilst population guidelines go some way to account for this, using characteristics such as age and sex, an increased understanding of the role of genetics in nutrient metabolism has highlighted the inadequacy of this 'one-size-fits-all' approach. The concept of tailoring nutritional advice based on clinical indicators, physiological states and nutrient intake is not novel. However, since the completion of the human genome project in $2003^{(8)}$, understanding of complex interactions between environmental factors such as diet and genes has progressed significantly.

The sequencing of the human genome and identification of interactions between genes and nutrients transformed the concept of personalised nutrition. Out of this emerged the nutrigenomic and nutrigenetic disciplines that aim to further investigate these interactions and, ultimately, improve the treatment and prevention of nutrition-related diseases ${ }^{(9-11)}$. Nutrigenomics explores the effect of specific nutrients on gene expression, whereas nutrigenetics aims to explore the effect that genetic variation has on the interaction between nutrients and disease ${ }^{(12)}$. Over 1000 genetic variants have been identified in the last decade ${ }^{(13)}$; as a result, the potential to individualise diets based on observed nutrient-gene interactions has increased. The role of nutritional genomics and genetics in utilising knowledge of nutrient-gene interactions to tailor dietary advice to this end is a promising field of research that could revolutionise the way we deliver dietary advice for the prevention of chronic nutrition-related diseases. It has been shown that tailoring weight-loss diets based on genotypic parameters significantly improves dietary responsiveness, with individuals placed on the 'correct diet for their genotype' losing two to three times more weight over a 12-month period than those on the 'incorrect diet $^{(14)}$. The concept of personalised nutrition has thus evolved to include combinations of genetic, phenotypic and dietary data to tailor an individual's diet and optimise nutritional status.

Current dietary advice exists in the form of basic population guidelines for healthy individuals and tailored guidelines for individuals at high risk of disease ${ }^{(15)}$. However, as highlighted by Rose, individuals considered to be at low risk of disease contribute to a greater number of cases of disease than those considered to be at high risk $^{(16)}$. This could be due to the greater population of 'healthy individuals' or as a result of underlying genetic variations that we do not currently assess in clinical practice. Targeting health promotion towards these individuals could therefore result in a greater reduction in disease prevalence and mortality than targeting those at high risk. Results from a population-based prospective cohort investigating CVD events between 1993 and 2007 showed that the greatest number of events occurred in the population group with the 'least risk'. Although the diabetic population were statistically more likely to experience an event, just sixty-nine (25.4\%) CVD events were recorded in the diabetic group whereas a total of $160(17.7 \%)$ events occurred in the hyperglycaemic group and 732 $(8.2 \%)$ events in the normoglycaemic group ${ }^{(17)}$. This suggests that prevention of CVD events in a high-risk group will have less impact on public health than targeted prevention in the lower-risk groups who display no or little symptoms of diabetes. A clear difficulty with this approach is that individuals with no obvious symptoms of disease (for example, high cholesterol) may be less likely to engage with personalised nutrition and disease prevention strategies. Individuals with one or two symptoms of disease (high cholesterol and/or central obesity) have been found to be more willing to undergo genetic testing in order to follow a personalised diet than those without ${ }^{(18)}$. It seems likely, therefore, that individuals with a 'lower risk' of disease such as those with few symptoms and/or risk markers of disease might be an ideal target group for nutrigenomic-based personalised nutrition interventions aimed at preventing chronic disease.

The success of nutrigenomic-based personalised nutrition in benefiting public health is dependent on both its ability to facilitate dietary change and consumer interest in the service. The greatest challenge for any nutrition intervention is motivating individuals to change their dietary behaviours and in order for nutrigenomic-based personalised nutrition to benefit public health over and above existing strategies, it must have a greater effect on behaviour change. At present, research surrounding the efficacy of this type of personalised nutrition is in its infancy, although insight into its success can be gained from survey-based research investigating willingness to engage with a nutrigenomic-based personalised nutrition service. Several papers have been published examining attitudes towards genetic testing for polygenic disease susceptibility; however, consumer acceptance of nutrigenomic-based personalised nutrition as a whole has been investigated in a limited number of qualitative studies. The present review will thus examine both current evidence for the acceptance of genetic testing and personalised nutrition, and its potential to motivate individuals to change their diet and lifestyle, in order to advise on directions for future research and to inform clinical practice.

Consumer acceptance will be investigated in terms of consumer willingness to engage with nutrigenomic-based personalised nutrition and factors affecting this decision. The results of this will provide insight into strategies that can be used to increase willingness to engage with personalised nutrition therapy that may be informed by nutrigenomics in future clinical practice, and directions for future research, by identifying potential gaps in knowledge. Delivery of nutrigenomic-based personalised nutrition and 
its potential impact upon motivation to change will also be examined using recent focus group research.

\section{Willingness to undergo genetic testing for polygenic diseases}

Many studies have been conducted in $\operatorname{Russia}^{(19)}$, Finland ${ }^{(20,21)}$, the $\mathrm{UK}^{(22)}$, the Netherlands ${ }^{(23,24)}$, Canada ${ }^{(25)}$ and the $\mathrm{USA}^{(26)}$ regarding attitudes towards genetic testing. Whilst genetic testing is becoming routine practice for the screening of high-penetrance genes such as the BRCA1 gene which is associated with a $65 \%$ cumulative risk of developing breast cancer by the age of 70 years $^{(27)}$, its use in the identification of polymorphisms that may predispose individuals to chronic disease is not well established and therefore may pose a significant barrier to the uptake of nutrigenomic-based personalised nutrition. The acceptance of genetic testing for hereditary diseases is thus widely accepted. Results from the 2002 Eurobarometer also showed that Europeans were accepting of this application of biotechnology. The perceived risk from genetic testing was slight, with individuals reporting that they felt the use of genetic testing in identifying diseases such as cystic fibrosis was both useful and had the potential to improve lifestyle ${ }^{(28)}$. However, it cannot be assumed that members of the public will feel the same about preventative testing for polygenic diseases.

In 2005, an omnibus opinion survey was conducted in six European countries to investigate attitudes towards genetic testing for disease prevention and personalised nutrition. In total, $66 \%$ of respondents indicated that they would be willing to undergo genetic testing. Further to this, $27 \%$ of respondents would do so for the purpose of following a diet for personalised nutrition. The age group found to be the most accepting were those aged 65 years or over. Encouragingly, the UK population was found to be the most accepting of genetic testing for the purpose of personalised nutrition, with women slightly more willing to undergo testing than men ${ }^{(18)}$. In a UK survey, by Sanderson et al. ${ }^{(22)}$, however, it was found that men were slightly more likely than women to say that they would undergo genetic testing to determine heart disease risk. Interest for testing was greatest in adults aged 46-60 years. In this survey, 69\% of respondents reported willingness to undergo genetic testing for the prevention of CVD. Respondents were significantly more interested in predicting susceptibility to heart disease than cancer; the author suggested this was because CVD is seen as more preventable than cancer ${ }^{(22)}$.

In 2008, Cherkas et al. ${ }^{(29)}$ conducted a non-representative survey of UK public interest in Internet-based personal genome testing (PGT) for diseases such as CVD and diabetes. PGT involves self-administered genetic swab testing, the results of which are interpreted by a clinician and fed back to the participant in electronic format. In the study, a total of $48 \%$ of respondents reported being likely to order a PGT test if it were free; 30\% of respondents were unlikely to order the test. It was found that younger individuals ( $<50$ years) and men were more willing to undergo genetic testing than older individuals and women ${ }^{(29)}$. Results from an Internet-based survey conducted by the Human Genetics Commission in 2003 saw less positive results, with over $60 \%$ of respondents indicating that they would be unlikely to use such a service. The majority of these respondents would, however, consider a test if offered by their doctor as part of a general check-up ${ }^{(30)}$.

Willingness to undergo genetic testing was greatest in a 2009 survey of public attitudes in Russia, with 85\% of respondents indicating that they would be willing to undergo genetic testing to determine their risk of developing preventable diseases. A total of $88.5 \%$ of these respondents also indicated that they would try to change bad habits, follow a recommended diet or take medications on the basis of these results if a high risk of disease were identified. Women were found to be more curious about their health and, thus, more supportive of personal genotyping; interest was greatest in the 25 to 39-year-old age category ${ }^{(19)}$. The Canadian public was also been found to be widely accepting of nutrigenomics in a series of focus groups conducted by Morin ${ }^{(25)}$, however, the sample was not representative of the population. This acceptance was echoed in a 2012 survey in which 83\% of Canadian respondents agreed that they would take a genetic test to encourage themselves to adopt a healthier lifestyle. A total of $75 \%$ also agreed that learning about their genetic makeup would affect what they ate ${ }^{(31)}$.

Interestingly, it was noted in a survey conducted in the Netherlands that participants responded more favourably to genetic testing when reference was given to the prevention of disease. Whilst the majority of respondents agreed with the statement 'I do not want to know what kind of diseases I could get in the future', 52\% then said they would if it were to prevent disease. A minority of Dutch respondents $(21 \%)$ did not want to know their risk of getting certain diseases ${ }^{(24)}$.

Willingness to undergo genetic testing for the prevention of disease therefore ranges from 48 to $85 \%$. This variation could be due to population characteristics, the time in which the studies were conducted, cultural differences or the phrasing used in each of the questions. As shown in the aforementioned study by Henneman et al. ${ }^{(24)}$, question phrasing can have a significant impact on participant response; thus it is difficult to compare study results as the questions asked were distinctly different. Despite these positive responses, however, it is unclear what the true uptake of genetic testing would be in these scenarios. It has been found that despite positive responses to the testing of high-penetrance genes in the literature, uptake is poor ${ }^{(32)}$; it has therefore been suggested that the likelihood of testing might be less with the lower-risk alleles implicated in chronic diseases ${ }^{(33)}$. In terms of targeting a certain sex or age group the results are inconsistent. 
Sanderson et $a l^{(22)}$ and Cherkas et $a l^{(29)}$ found men to be more likely to undergo genetic testing whereas Stewart-Knox et al. ${ }^{(18)}$ and Makeeva et al. ${ }^{(19)}$ found that women were more likely to undergo genetic testing. Stewart Knox et al. ${ }^{(18)}$ and Sanderson et al. ${ }^{(22)}$ found that older adults, aged 65+ and 46-60 years, respectively, were more accepting of genetic testing whereas Cherkas et $a l .{ }^{(29)}$ and Makeeva et $a .^{(19)}$ found that younger adults, aged $<50$ and 25-39 years, respectively, were more accepting ${ }^{(18,19,22,29)}$.

\section{Factors influencing willingness to undergo genetic testing}

To understand the reasons for the proposed uptake of genetic testing and how to increase it, it is important to understand individual motivations behind the decision to undergo genetic testing. Several factors were identified in the aforementioned papers that influenced an individual's willingness to undergo genetic testing for polygenic disease risk.

In the surveys by Makeeva et al. ${ }^{(19)}$ and Stewart-Knox et $a l{ }^{(18)}$ health was identified as the primary motivator for undergoing genetic testing, with $38 \%$ of Russian respondents and $47.4 \%$ of European respondents giving answers that related to health and dietary change. Reasons for undergoing genetic testing in the later survey were provided in response to an open-ended question. The health-related responses given included: getting healthier and/or reducing disease risk (18.8\%), making dietary changes $(14.4 \%)$, having a health problem $(7.6 \%)$ or having a family history of disease (6\%). Makeeva et al. ${ }^{(19)}$ found that a doctor's recommendation was the second most frequently chosen motivator for willingness to undergo genetic testing; this was chosen more so in the older age category. A total of $17 \%$ of Russian respondents and $10.7 \%$ of European respondents listed curiosity as their motivation to take a genetic test. Makeeva et al. ${ }^{(19)}$ found that men listed curiosity as their motivation more frequently than women and that curiosity decreased with age $^{(18,19)}$. In the Dutch survey by Henneman et al. ${ }^{(24)}$ a third of respondents agreed that they were curious about their genetic makeup.

In the study by Stewart-Knox et al. ${ }^{(18)}$ respondents were also required to self-report the following symptoms of the metabolic syndrome: high blood pressure, high blood cholesterol levels, high blood glucose levels, a high proportion of fat around the middle and increased perceived stress. It was found that individuals who were willing to undergo genetic testing were 1.35 times more likely to report central obesity and 1.27 times more likely to have high cholesterol. In addition, significantly more of these were willing to undergo genetic testing to follow a personalised diet as opposed to taking the test for general interest ${ }^{(18)}$.

The most common reason for not undergoing genetic testing to determine preventable polygenic disease risk in the Russian population was cost ( $41 \%)$; this was followed by time concerns $(20 \%)$ and fear of results $(14 \%)^{(19)}$. Additional reasons identified in the European population included: scepticism, mistrust, unproven efficacy and not wanting to know. Some participants felt healthy eating was enough, or did not want to change their diet ${ }^{(18)}$. Cherkas et al. ${ }^{(29)}$ similarly found health to be the most important determinant of willingness to undergo genetic testing, with $93 \%$ of those respondents likely to take a PGT agreeing that they would do so to encourage them to lead a healthier lifestyle. Younger individuals and women were more likely to endorse this reason than older individuals and men. Support for alternative reasons for taking a PGT were as follows: 'to learn more about myself' (86\%), 'to convey genetic risk to my children' $(80 \%)$, 'so a doctor can monitor my health more closely' (79\%) and 'to assist in future financial planning' $(50 \%)^{(29)}$.

In the study by Henneman et al. ${ }^{(24)}$ it was found that individuals who responded favourably towards genetic testing were more likely to have personal experience of a genetic disease, believe that they would benefit from genetic testing and/or believe the knowledge of the genetic background of disease would help to increase an individual's lifespan. Sanderson et al. ${ }^{(22)}$ found than individuals with at least one close family member with heart disease were more likely to express interest in genetic testing for preventable diseases.

Adámková et al. ${ }^{(34)}$ have proposed that the key reason for the varied response seen in willingness to participate in genetic testing for polygenic disease risk is a lack of knowledge and understanding, suggesting that it is crucial to enhance public knowledge of genetic testing and its applications. Morren et al. ${ }^{(23)}$ also suggested that the decision to undergo genetic testing was dependent on an individual's knowledge and perceptions about genetic research. It was found that chronically diseased individuals with a higher perceived knowledge of genetic testing had a more positive attitude than those with less awareness of the medical possibilities of genetic testing, such as the prevention and early treatment of a disorder. Contrary to this, Henneman et al. ${ }^{(24)}$ found no association between participant attitudes and genetic knowledge. The author suggests that more information would therefore not necessarily result in increased interest in genetic testing. In focus groups by Morin ${ }^{(25)}$ it was found that the majority of the public was unfamiliar with the term 'nutrigenomics' and only half of the dietitians, nutritionists and naturopaths were aware of it, with few participants able to offer correct definitions of the term. Participants related the term 'personalised nutrition' to healthy eating advice and individually tailored plans yet only a few health care professionals (HCP) made the link with genetic profiling ${ }^{(25)}$

Factors affecting acceptance of nutrigenomic-based personalised nutrition have also been investigated more recently by Ronteltap et al. ${ }^{(35)}$. The study used an innovative research approach to determine consumer preference using a systematically varied design based on film scenes. 
A total of 416 participants took part in the study, and freedom of choice was found to be the most important driver of consumer acceptance. Clear consumer benefits (relating to health improvements), agreement amongst experts, ease of implementation, cost and peer support were also important determinants of consumer acceptance.

It therefore appears that health may be the most important determinant of undergoing genetic testing ${ }^{(18,19,29)}$. At present, personalised nutrition services tend to target individuals 'wanting to lose weight' or have a 'healthier lifestyle, (36). Personal experience of disease, such as having a family member with a disease, appears important for increasing motivation to undergo gene testing ${ }^{(18,24)}$ as it has been found that individuals with a heightened perceived susceptibility to disease show more interest in genetic testing ${ }^{(37)}$. Clear consumer benefits are also an important determinant in both motivation to change and consumer acceptance ${ }^{(24,38)}$. Current evidence therefore suggests that individuals at a low risk of disease such as those with few disease symptoms would be interested in a nutrigenomic-based personalised nutrition service.

\section{Perceived benefits}

It is important to investigate the perceived risks and benefits of genetic testing for personalised nutrition in order to address potential barriers to a nutrigenomic service and facilitate successful commercialisation to consumers. As mentioned, clear consumer benefits have been identified as a key determinant of acceptance ${ }^{(35)}$. Several other benefits have also been identified which include: assisting in the prevention of disease, reducing health care costs and improving motivation to change. Encouragingly, Nielsen \& El-Sohemy ${ }^{(31)}$ reported that individuals found genotypebased advice more understandable and more useful in relation to their diet compared with general dietary advice. They also found the genotype-based advice more enjoyable to read.

Morin ${ }^{(25)}$ found that members of the Canadian public were open to the concept of nutritional genomics, identifying its potential benefit in assisting with the early diagnosis and prevention of disease. The majority of Dutch respondents (43\%) in the survey by Henneman et al. ${ }^{(24)}$ believed that knowledge of the genetic background of disease could increase an individual's lifespan. A quarter of the respondents also believed that if they knew their genetic risk they would be able to control their lives more. In the Russian population $81 \%$ of respondents were in agreement with this statement ${ }^{(19)}$. This is important to note, as the core aim of health promotion is to 'enable people to increase control over, and to improve their health'(39). A total of $2.8 \%$ of European Union respondents in the survey by Stewart-Knox et al. ${ }^{(18)}$ listed 'taking control' as their reason for undergoing genetic testing. As well as encouraging healthier food consumption, nutrigenomics may also reduce health care costs associated with disease treatment ${ }^{(38)}$. The majority of respondent in the study by Aro et $a l .{ }^{(20)}$ agreed that genetic testing was acceptable, because society could save on the costs of treating diseases. On account of its potential benefits to society, $69 \%$ of respondents in the survey by Makeeva et al. ${ }^{(19)}$ felt that genetic testing should be extensively promoted. Respondents in this survey were also asked to select diseases that they deemed most valuable for genetic testing. Oncological diseases were seen as the most suitable (16.5\%), followed by CVD (15.8\%) and diabetes $(11 \cdot 0 \%)^{(19)}$.

\section{Motivation to change behaviour}

As stated by Ronteltap \& van Trijp ${ }^{(40)}$, nutrigenomic-based personalised nutrition will only contribute to quality of life if consumers are motivated to follow the individual recommendations on food intake. Motivation to change behaviour is determined by perceptions of self-efficacy and outcome expectations, that is, the belief in one's ability to change a given behaviour and whether this alteration will achieve the expected outcome ${ }^{(41)}$. The potential for nutrigenomic-based personalised nutrition to increase motivation to change has been questioned by some researchers ${ }^{(42,43)}$; however, there is little evidence to support this hypothesis. Several possible motivational outcomes have been considered: Joost et al. ${ }^{(43)}$ suggested that individuals identified as having a higher disease risk through genetic testing may be more motivated to comply with a dietary intervention; however, it was also noted that knowledge of a genetic predisposition may result in a fatalistic attitude and reduced compliance. Furthermore, Hunter et al. ${ }^{(44)}$ reasoned that a negative result may lead to reduced motivation as individuals become reassured that they will not develop disease. Results from a pilot investigation by Harvey-Berino et al. ${ }^{(45)}$ into the effect of genetic testing on confidence in ability to lose weight found that a positive obesity gene status did not adversely affect participants' selfefficacy. The author found that participants reported increased confidence in their ability to lose weight, regardless of their obesity gene status ${ }^{(45)}$.

Makeeva et $a l .{ }^{(19)}$ reported that women were more positive about behaviour change as a result of genetic testing than men, and that positivity about change was not dependent on health status. In focus groups conducted in Ireland, both dietitians, and obese and diabetic patients suggested that nutritional genomics might have a positive impact on behaviour change and health improvements. However, whilst dietitians recognised the potential of gene testing to motivate patients, they doubted whether behaviour change would be achieved. The morbidly obese and type 2 diabetic subjects in this study also expressed concerns that, whilst the potential for motivation was high, change was not guaranteed ${ }^{(46)}$. In a study conducted on patients with a history of weight loss 
failures, it was found that nutrigenetic test screening increased compliance and resulted in longer-term BMI reductions when compared with standard weight-loss advice $^{(47)}$. This was supported by a study that reported that individuals at a high genetic risk of obesity had greater intentions to eat a healthy diet compared with 'averagerisk' participants. However, it was also shown that the high-risk individuals had lower perceived behavioural control over their eating ${ }^{(48)}$ and hence felt less able to change their dietary habits.

\section{Perceived risks}

In addition to these perceived benefits, several concerns have been raised by both researchers and consumers regarding privacy, the delivery of information and fear of results.

\section{Delivery of information}

Nutrigenomic services may be provided in the clinical setting via face-to-face interaction with $\mathrm{HCP}$ or via the Internet using direct-to-consumer (DTC) tests. While DTC genetic testing is a growing phenomenon in the USA ${ }^{(49)}$, it has seen less success in the UK. There is a large evidence base regarding the ethical, legal and social implications of nutrigenomics and DTC testing although, as this is mostly theoretical, it is unclear if the concerns raised in these studies will be echoed by the general public. However, this research provides insight into some of the potential concerns that individuals may have about nutrigenomicbased personalised nutrition, particularly when delivered via the Internet using DTC testing.

It has been noted that consumers can be suspicious about commercial interests of industries ${ }^{(40)}$. Both Canadian HCP and members of the public were concerned that genetic tests might be overly expensive ${ }^{(25)}$. Nutrition services based on genetic information have been found to be the most highly priced ${ }^{(36)}$. Cost was also the most common reason for not undergoing genetic testing in the Russian population $^{(19)}$. When survey respondents were told that the PGT would cost $£ 250$ in the study by Cherkas et al., the percentage of individuals willing to take the test dropped from $48 \%$ to $5 \%$, indicating that cost was a critical factor in uptake. Willingness to undergo genetic testing in this study was the lowest out of all the studies reviewed; this may have been due to the use of an Internet-based PGT model ${ }^{(29)}$. In focus group studies some members of the Canadian public expressed concern that private companies running nutrigenomic services may be motivated by financial gain as opposed to health promotion ${ }^{(25)}$. This population group expressed a clear preference for one-to-one testing with a HCP. Amongst Dutch patients with chronic disease, the greatest preference for information about genetic testing was a general practitioner (50.6\%) followed by information sheets or another medical professional $^{(23)}$. However, not only are nutrigenomics services not currently offered by the National Health Service in the UK, but HCP have been found to have a lack of knowledge and education regarding the subject and genetic testing in general ${ }^{(19,50)}$. Following a series of focus groups conducted in Canada with physicians, pharmacists, dietitians, naturopaths and nutritionists, Weir et al. also concluded that: 'health care professionals are not yet ready to incorporate nutrigenetic testing into clinical practice $^{, 51)}$. Whelan et al. ${ }^{(52)}$ similarly found that knowledge of gene-diet interactions was poor amongst dietitians in the UK, highlighting the need for increased education and training in this group. A key finding in the systematic review by Scheuner et al. ${ }^{(53)}$ was that the primary care workforce felt underprepared to integrate genomic medicine into clinical practice. It is important to consider HCP knowledge and attitudes towards nutrigenomics, as this may make an impact upon consumers' confidence in the service and its successful introduction. The European Nutrigenomics Organisation has gone some way to address this issue by organising training courses for HCP.

In addition to the way in which nutrigenomics advice is provided, consumers have expressed concerns about the manner in which it is introduced. The majority of Dutch respondents $(57 \%)$ in the survey by Henneman et al. ${ }^{(24)}$ were worried that individuals would be forced to undergo genetic testing, with only $11 \%$ of respondents agreeing that genetic testing should be promoted. This is in line with the results of another study that identified freedom of choice as the most important driver of consumer acceptance ${ }^{(35)}$.

\section{Privacy}

Privacy and the misuse of genetic information may also pose a significant risk for many consumers considering genetic testing. In a paper regarding the ethical, legal and social issues in nutrigenomics, it is noted that in the USA, it is possible that insurance companies would have an interest in DNA results as a means of risk rating clients for policy quotes ${ }^{(50)}$. Morren et al. ${ }^{(23)}$ found that just under half of the respondents who took part in the survey agreed that they would worry about the effect that DNA testing might have on taking out insurance policies. Approximately one-third were also worried that it would make an impact on finding a job ${ }^{(23)}$. When exposed to a mock Internet-based personalised nutrition service, HCP and Canadian consumers expressed concerns about potential breaches of privacy ${ }^{(25)}$. However, Castle \& Ries ${ }^{(50)}$ suggest that DTC testing offers greater privacy protection than services provided by health services, as results are not stored in general medical notes, thus reducing the availability of the data to third parties. Stewart-Knox et al. ${ }^{(18)}$ described a relative lack of concern amongst the British public regarding privacy; however, unlike the questionnaire used in the study by Morren et al. ${ }^{(23)}$ where participants were asked to agree or disagree with a series of statements, 
respondents were asked to choose just one of eight reasons for accepting or rejecting nutrigenomic intervention. It is therefore possible that participants were concerned about privacy, yet not to the extent that it dissuaded them from undergoing genetic testing. Over half of respondents chose the item: 'it is good to know if you are genetically at risk', with only $1.2 \%$ being primarily worried about use of information by insurers and $0.9 \%$ by employers. Of the respondents, $1 \%$ were most worried about how the information would be used by the police ${ }^{(18)}$. In recent recommendations from the European Society of Human Genetics it was advised that 'governments proceed with additional non-discrimination legislation in relation to genetic information in order to ensure that both employers and insurance companies use genetic information in a responsible and ethically justified manner, ${ }^{(54)}$.

\section{Fear of result outcomes}

In addition to concerns regarding the process of genetic testing, consumers are also likely to have fears regarding test outcomes. A total of $14 \%$ of Russian respondents gave fear of results as their reason for not undergoing genetic testing, and $4 \%$ gave fear of treatment. The majority of Dutch participants were not fearful of genetic test results; however, $41.5 \%$ of respondents said they would not have the test done if the disease could not be treated ${ }^{(23)}$. HCP and members of the Canadian public were also concerned that results could cause anxiety for users or that they might be inaccurate or unclear ${ }^{(25)}$. A systematic review into the perceived risks, and psychological and behavioural impacts of genetic testing for hereditary cancers and Alzheimer's disease found that, overall, genetic testing had no impact on distress, anxiety or depression in either carriers or non-carriers of gene variants. However, most of the studies reviewed utilised extensive pre- and posttest counselling, which is likely to have had an impact on perceived risk ${ }^{(55)}$.

Concerns about genetic testing have also been raised regarding the impact of result outcomes on the wider society. A total of $48 \%$ of Russian respondents believed that publicly available genetic testing could lead to discrimination and $44 \%$ of Dutch participants were worried that genetic testing may result in a society where disabled individuals were not accepted. The majority of respondents also felt that genetic testing could lead to discrimination ${ }^{(24)}$. Finnish respondents were equally concerned that such results may lead to discrimination ${ }^{(20)}$. Another issue raised with genetic testing in general is whether or not individuals should inform family members of their results. This is perhaps more relevant in the testing of highpenetrance genes where there is a strong hereditary link, but the topic has been covered in the context of testing for multifactorial diseases. In the study conducted by Morren et $a l .^{(23)}$, it was found that the majority of Dutch patients with chronic disease felt that family members would need to know the result.

\section{Discussion}

Nutrigenomic-based personalised nutrition has the potential to benefit public health, provided that it motivates individuals to change their dietary behaviours, and that consumers are willing to engage. Resistance to the use of genetic information to tailor individual nutritional advice poses a potential barrier in this uptake of nutrigenomic-based personalised nutrition. Whilst no firm conclusions can be reached regarding the impact of sex, age and knowledge on acceptance of genetic testing, health concerns and clear consumer benefits have been established as key determinants of uptake. Given the empirical nature of the studies reviewed it is impossible to accurately predict public response to this application of genetic testing, which has thus far seen limited commercial success. However, the studies reviewed indicate that a significant proportion of the public would be interested in undergoing genetic testing for the prevention of chronic disease. Individuals with a heightened perceived susceptibility to disease, who express few disease risk markers (for example, high cholesterol, obesity, family history), might be an ideal target group for nutrigenomic-based personalised nutrition, given that they contribute significantly to disease events and are more willing to engage with personalised nutrition. A caveat of this approach is that it relies on individuals being aware of the symptoms of disease; a recent cross-sectional survey found that $48 \%$ of the public were unable to name one symptom of diabetes ${ }^{(56)}$.

The questionnaires used in the studies vary in their application of genetic testing; some relate to chronic diseases in general, whereas others relate to specific examples of disease. The delivery of the genetic tests also varies from PGT to one-to-one interaction. Thus far it appears that a free service involving one-to-one contact with HCP is the most preferred method of nutrigenomic-based personalised nutrition delivery, although this business model does not exist at present: in a recent publication by Ronteltap et $a l^{(36)}$ it was found that companies that are currently using genotypic data to tailor advice deliver their services solely via the Internet using one-to-one online relationships. In total, the researchers uncovered nine archetypes of personalised nutrition, one of which involved genetic testing. Compared with face-to-face consultation, Internet delivery of nutrigenomic-based personalised nutrition may be more financially viable, especially given the potential of computer-based algorithms. A potential reason for the lack of one-to-one delivery of nutrigenomic-based personalised nutrition is lack of knowledge amongst HCP. Future research should focus on the most effective delivery of nutrigenomic-based personalised nutrition, comparing the effect of different methods on dietary change and disease prevention. The ability of nutrigenomic testing to 
facilitate behaviour changes in individuals is the greatest barrier to the success and efficacy of nutrigenomic-based personalised nutrition, and at present the evidence is limited and predominantly associated with weight-loss interventions. It is therefore crucial to investigate this outcome further in order to predict its benefit in healthcare. The 7th Framework Programme for European Union Research (FP7) study, Food4Me, aims to investigate both the consumer acceptance and application of personalised nutrition. Food $4 \mathrm{Me}$ is the first study to investigate the effectiveness of personalised nutrition and is designed to mimic a fully Internet-delivered, personalised nutrition service $^{(57)}$. The efficacy of personalised nutrition advice to motivate dietary change will be investigated at the levels of diet, phenotype and genotype, providing muchneeded evidence for the potential impact of personalised nutrition on public health.

There is a need for HCP and practitioners to increase their nutrigenomic knowledge to ensure that healthcare services are able to cope with this degree of tailoring in the future, in line with the anticipated advances in dietary prevention and treatment of NCD. Research into the use of nutrigenomic-based personalised nutrition to improve treatments in patient groups such as diabetics and individuals with cancer is on-going ${ }^{(26,58)}$. Grant et al. ${ }^{(26)}$ found that physicians would be more likely to recommend genetic testing for medication profiling $(47.7 \%)$ than for predicting genetic risk of diabetes (21.7\%), although they were more positive about the effect that predictive testing would have on lifestyle change. Ronteltap et al. ${ }^{(36)}$ have also suggested that future research should focus on establishing continuous relationships with individuals using goal achievement to ensure the long-term success of personalised nutrition in reducing disease risk and healthcare costs; it has been estimated that a $1 \%$ reduction in relative risk of CVD in England and Wales could save the National Health Service $£ 30$ million per year ${ }^{(59)}$.

\section{Conclusions}

Individuals with personal experience of chronic disease, such as those with symptoms of disease, have a heightened perceived susceptibility to disease and could be ideal candidates for a nutrigenomic-based personalised nutrition service. The ability of nutrigenomic testing to facilitate behaviour change in individuals is by far the greatest barrier to the success and efficacy of the intervention; it is crucial to investigate this outcome further in order to assess its benefit in healthcare. Several risks have been identified in relation to genetic testing. It is important to adequately address these and ensure effective communication to allay individuals' fears when designing a nutrigenomicbased personalised nutrition service. There is also a need for clear and realistic intervention outcomes to ensure that potential users engage with the service and are motivated to change diet-related behaviours. It is unclear whether public education regarding genetic testing for multifactorial diseases will help to improve uptake of a nutrigenomic-based personalised nutrition service or whether genetic counselling would be beneficial.

The results of the present review suggest that individuals with symptoms of disease are likely to engage with a nutrigenomic-based personalised nutrition service, provided that it is delivered in an acceptable manner and that privacy concerns are addressed. In the future it would be useful to conduct further pan-European studies to determine whether the differences identified thus far are as a result of population differences or study design. This, in addition to further scenario-based research on service delivery and barriers to uptake in the UK, will help to define an appropriate target population and further predict the success of nutrigenomic-based personalised nutrition in reducing the burden of NCD.

\section{Acknowledgements}

This research received no specific grant from any funding agency in the public, commercial or not-for-profit sectors.

R. F. conducted the review of the literature and drafted the manuscript. J. A. L and L. T. B. contributed significantly to the drafting of the manuscript. All authors made a critical review of the draft.

There are no conflicts of interest.

\section{References}

1. World Health Organization (2001) Diet, Physical Activity and Health, Documents A55/16 and A55/16 Corr. 1). Geneva: WHO.

2. Allender S, Scarborough P, Peto V, et al. (editors) (2008) European Cardiovascular Disease Statistics, 2008 Edition. In European Cardiovascular Disease Statistics, p. 112 Oxford: University of Oxford.

3. Alwan A (2011) Global Status Report on Noncommunicable Diseases 2010. Geneva: WHO.

4. Kreuter MW \& Wray RJ (2003) Tailored and targeted health communication: strategies for enhancing information relevance. Am J Health Behav 27, S227-S232.

5. Marantz PR (2010) Rethinking dietary guidelines. Crit Rev Food Sci Nutr 50, 17-18.

6. Hippisley-Cox J, Coupland C, Vinogradova Y, et al. (2007) Derivation and validation of QRISK, a new cardiovascular disease risk score for the United Kingdom: prospective open cohort study. Br Med J 335, 136.

7. Hesketh J, Wybranska I, Dommels Y, et al. (2006) Nutrientgene interactions in benefit-risk analysis. Br J Nutr $\mathbf{9 5}$, $1232-1236$.

8. National Institutes of Health (2003) NIH News - international consortium completes human genome project: all goals achieved; new vision for genome unveiled. http://www.nih. gov/news/pr/apr2003/nhgri-14.htm (accessed December 2011).

9. van Ommen V (2007) Personalized nutrition from a health perspective: luxury or necessity? Genes Nutr 2, 3-4.

10. Kussmann M, Raymond F \& Affolter M (2006) OMICS-driven biomarker discovery in nutrition and health. $J$ Biotechnol 124, 758-787. 
11. Kaput J \& Rodriguez RL (2004) Nutritional genomics: the next frontier in the postgenomic era. Physiol Genomics 16, $166-177$.

12. Ordovas JM \& Mooser V (2004) Nutrigenomics and nutrigenetics. Curr Opin Lipidol 15, 101-108.

13. Mutch D, Wahli W \& Williamson G (2005) Nutrigenomics and nutrigenetics: the emerging faces of nutrition. FASEB $J$ 19, $1602-1616$.

14. Dopler Nelson M, Prabakar P \& Kondragunta V (2010) Genetic phenotypes predict weight loss success: the right diet does matter. In 50th Cardiovascular Disease Epidemiology and Prevention and Nutrition, Physical Activity, and Metabolism 2010, abstract 735. San Francisco: American Heart Association.

15. Department of Heath (2008) Putting Prevention First - Vascular Checks: Risk Assessment and Management. London: Department of Health.

16. Rose G, Khaw K \& Marmot M (2008) Rose's Strategy of Preventative Medicine. Oxford: Oxford University Press.

17. Simmons RK, Coleman RL, Price HC, et al. (2009) Performance of the UK Prospective Diabetes Study risk engine and the Framingham risk equations in estimating cardiovascular disease in the EPIC-Norfolk cohort. Diabetes Care 32, 708-713.

18. Stewart-Knox BJ, Bunting BP, Gilpin S, et al. (2008) Attitudes toward genetic testing and personalised nutrition in a representative sample of European consumers. Br J Nutr 101, 982-989.

19. Makeeva OA, Markova VV \& Puzyrev VP (2009) Public interest and expectations concerning commercial genotyping and genetic risk assessment. Pers Med 6, 329-341.

20. Aro AR, Hakonen A, Hietala M, et al. (1997) Acceptance of genetic testing in a general population: age, education and gender differences. Patient Educ Couns 32, 41-49.

21. Toiviainen H, Jallinoja P, Aro AR, et al. (2003) Medical and lay attitudes towards genetic screening and testing in Finland. Eur J Hum Genet 11, 565-572.

22. Sanderson SC, Wardle J, Jarvis MJ, et al. (2004) Public interest in genetic testing for suceptability to heart disease and cancer: a population-based survey in the UK. Prev Med 39, 458-464.

23. Morren M, Rijken M, Baanders AN, et al. (2007) Perceived genetic knowledge, attitudes towards genetic testing, and the relationship between these among patients with a chronic disease. Patient Educ Couns 65, 197-204.

24. Henneman L, Timmermans DRM \& Wal GVD (2006) Public attitudes toward genetic testing: perceived benefits and objections. Genet Test 10, 139-145.

25. Morin K (2009) Knowledge and attitudes of Canadian consumers and health care professionals regarding nutritional genomics. OMICS 13, 37-41.

26. Grant R, Hivert M, Pandiscio J, et al. (2009) The clinical application of genetic testing in type 2 diabetes: a patient and physician survey. Diabetologia 52, 2299-2305.

27. Antoniou A, Pharoah P, Narod S, et al. (2003) Average risks of breast and ovarian cancer associated with BRCA1 or BRCA2 mutations detected in case series unselected for family history: a combined analysis of 22 studies. Am J Hum Genet 72, 1117-1130.

28. Gaskell G, Allum N \& Stares S (2003) Europeans and biotechnology in 2002, Eurobarometer 58.0 p. 44. http:// ec.europa.eu/public_opinion/archives/ebs/ebs_177_en.pdf (accessed February 2013).

29. Cherkas LF, Harris JM, Levinson E, et al. (2010) A survey of UK public interest in Internet-based personal genome testing. PLoS One 5, e13473.

30. Human Genetics Commission (2003) Genes Direct: Ensuring the Effective Oversight of Genetic Tests Supplied Directly to the Public. London: Department of Health.
31. Nielsen DE \& El-Sohemy A (2012) A randomized trial of genetic information for personalized nutrition. Genes Nutr $\mathbf{7}$, $559-566$

32. Meiser B, Kasparian NA, Mitchell PB, et al. (2008) Attitudes to genetic testing in families with multiple cases of bipolar disorder. Genet Test 12, 233-243.

33. Makeeva OA, Markova VV, Roses AD, et al. (2010) An epidemiologic-based survey of public attitudes towards predictive genetic testing in Russia. Pers Med 7, 291-300.

34. Adámková V, Veleminský M, Zimmelová P, et al. (2009) Volunteer's willingness to genetic testing - lack of the understanding of the matter. Physiol Res 58, S53-S54.

35. Ronteltap A, van Trijp JCM \& Renes RJ (2009) Consumer acceptance of nutrigenomics-based personalised nutrition. BrJ Nutr 101, 132-144.

36. Ronteltap A, van Trijp H, Berezowska A, et al. (2013) Nutrigenomics-based personalised nutritional advice: in search of a business model? Genes Nutr, 8, 153-163.

37. Bosompra K, Flynn BS, Ashikaga T, et al. (2000) Likelihood of undergoing genetic testing for cancer risk: a populationbased study. Prev Med 30, 155-166.

38. Ronteltap A, Van Trijp J, Renes R, et al. (2007) Consumer acceptance of technology-based food innovations: lessons for the future of nutrigenomics. Appetite 49, 1-17.

39. World Health Organization (1986) Ottawa Charter for Health Promotion. Geneva: WHO.

40. Ronteltap A \& van Trijp H (2007) Consumer acceptance of personalised nutrition. Genes Nutr 2, 85-87.

41. Strecher VJ, DeVellis BME, Becker MH, et al. (1986) The role of self-efficacy in achieving health behavior change. Health Educ Behav 13, 73-92.

42. Sanderson SC, Wardle J \& Humphries SE (2008) Public health genomics and genetic test evaluation: the challenge of conducting behavioural research on the utility of lifestyle-genetic tests. J Nutrigenet Nutrigenomics 1, 224-234.

43. Joost H, Gibney M, Cashman K, et al. (2007) Personalised nutrition: status and perspectives. Br J Nutr 98, 26-31.

44. Hunter DJ, Khoury MJ \& Drazen JM (2008) Letting the genome out of the bottle - will we get our wish? $N$ Engl J Med 358, 105-107.

45. Harvey-Berino J, Gold EC, West DS, et al. (2001) Does genetic testing for obesity influence confidence in the ability to lose weight? A pilot investigation. J Am Diet Assoc 101, 1351-1353.

46. McMahon OC, McConnon A, McKenzie K, et al. (2010) An exploration of the use of personalised nutrition among potential end users of the concept. Proc Nutr Soc 69, $\mathrm{E} 357$

47. Arakadianos I, Valdes AM, Marinos E, et al. (2007) Improved weight management using genetic information to personalize a calorie controlled diet. Nutr J 6, 29.

48. Frosch DL, Mello P \& Lerman C (2005) Behavioral consequences of testing for obesity risk. Cancer Epidemiol Biomarkers Prev 14, 1485-1489.

49. Hogarth S, Javitt G \& Melzer D (2008) The current landscape for direct-to-consumer genetic testing: legal, ethical, and policy issues. Annu Rev Genomics Hum Genet 9, 161-182.

50. Castle D \& Ries NM (2007) Ethical, legal and social issues in nutrigenomics: the challenges of regulating service delivery and building health professional capacity. Mutat Res $\mathbf{6 2 2}$, $138-143$

51. Weir M, Morin K, Ries N, et al. (2010) Canadian health care professionals' knowledge, attitudes and perceptions of nutritional genomics. BrJ Nutr 104, 1112-1119.

52. Whelan K, McCarthy S \& Pufulete M (2008) Genetics and diet-gene interactions: involvement, confidence and knowledge of dietitians. BrJ Nutr 99, 23-28. 
53. Scheuner MT, Sieverding P \& Shekelle PG (2008) Delivery of genomic medicine for common chronic adult diseases. $J \mathrm{Am}$ Med Assoc 299, 1320-1334.

54. van El CG \& Cornel MC (2011) Genetic testing and common disorders in a public health framework. Eur J Hum Genet 19, 377-381.

55. Heshka JT, Palleschi C, Howley H, et al. (2008) A systematic review of perceived risks, psychological and behavioral impacts of genetic testing. Genet Med 10, 19-32.
56. Jackson D, Wills R, Davies J, et al. (2009) Public awareness of the symptoms of diabetes mellitus. Diabet Med 8, 971-972.

57. Walsh M \& Kuhn S (2012) Developments in personalised nutrition. Nutr Bull 37, 380-383.

58. Mathers JC (2007) Overview of genes, diet and cancer. Genes Nutr 2, 67-70.

59. Barton P, Andronis L, Briggs A, et al. (2011) Effectiveness and cost effectiveness of cardiovascular disease prevention in whole populations: modelling study. BMJ 343, d4044. 